\title{
Malignant Metastatic Insulinoma in a Dog
}

Ozlem Ozmen' \& Yusuf Sinan Sirin ${ }^{2}$

\begin{abstract}
Background: Insulinomas are tumors of the pancreatic islet beta cells that secreting insulin. They are malignant neoplasms and rarely seen in dogs. Because insulinomas secrete excessive insulin and hypoglycemia occur. The most common clinical symptoms are seizures, extreme weakness, and other neurological abnormalities. The tumors typically release insulin episodically, with clinical signs being seen intermittently as a result. These are most often associated with prolonged starvation or prolonged periods of exertion. Insulinoma occurs most often in middle-aged and older dogs, averaging nine years in age. They affect both sexes and are more likely in larger breeds. The most commonly afflicted breeds are German Shepherds, Irish Setters, Boxers, Golden Retrievers and Terriers.

Case: In this study, a case of malignant insulinoma with multiorgan metastasis in a 7-year-old hunting dog that brought to the clinic with complaints of constipation and inappetence were evaluated by clinical, cytological, histopathological and immunohistochemical findings. Owner stated that the animal suffers from constipation for 4 days, but no vomitus or nervous symptoms were present. Clinical efforts not solved the constipation. For to the definitive diagnosis the dog decided to underwent laparotomy. After a midline abdominal approach multiple whitish, hard and irregular metastatic tumoral masses were observed in different abdominal organs. Because of the poor prognosis and according to owner's acceptance the dog euthanatized at the same time. At necropsy, a bloody serous fluid approximately $500 \mathrm{~mL}$ was found in the abdominal cavity. The intestines were hyperemic and hemorrhagic in some areas. A hemorrhagic $9 \times 5 \times 3 \mathrm{~cm}$ in diameter mass was observed on the pancreas. Mesenterial adhesions were formed due to mass between duodenum and jejunum. The mass was moderately hard and cut surface was bloody, necrosis was observed in some areas. At the gross examination, whitish color, various sized and multiple metastatic masses were noted in spleen, liver, lymph nodes and lungs. Before formalin fixation, impression smears were made from the pancreatic mass. In cytological preparations of the lesion, epithelial cells in different shapes and sizes were observed. At the histopathological examination, the tumor mass consisted of anaplastic, pleomorphic cells that have pale cytoplasm, vesicular nucleus and prominent nucleolus. Diffuse necrotic areas were noted in the mass. Similar metastatic masses were seen in liver, spleen, lungs and kidneys. The tumor cells were positive for insulin and negative for glucagon immunohistochemically.

Discussion: Insulinomas generally caused nervous symptoms due to hypoglycemia. Metastasis may occur in some cases and generally lymph node metastasis occurs. Spleen metastasis are very rare in insulinoma cases. The mean age of the occurrence of insulinoma is generally older than 9-year-old. In this case, a case of malignant insulinoma with multiorgan metastasis and caused constipation due to intestinal adhesions in a dog was presented with clinical and pathological findings. Best of the author's knowledge insulinoma as a cause of constipation not previously reported in a dog. The aim of this study was to report a case of metastatic insulinoma without neurological symptoms but constipation in a 7 years old hunting dog by clinical, pathological, cytological and immunohistochemical findings.
\end{abstract}

Keywords: Malignant insulinoma, constipation, cytology, histopathology, immunohistochemistry, dog. 


\section{INTRODUCTION}

Occurrence of primary pancreatic neoplasm is relatively rare in dogs $[5,11,13]$. The most of the pancreatic neoplasms are derived from acinar or pancreatic duct epithelial cells of exocrine pancreas. The exocrine pancreatic neoplasm is usually diagnosed as malignant tumors. Transcoelomic, local invasive and long distant metastasis are usually occurred before the onset of clinical signs $[1,3,4,6]$.

Clinical symptoms are secondary to hypoglycemia, and commonly include neuromuscular findings. The severity of clinical signs depends upon the duration and severity of hypoglycemia and the rate at which the blood glucose concentration falls. When fasting hypoglycemia is recurrent or persistent, adaptation permits tolerance of extremely low blood glucose levels without obvious clinical signs. Generally, clinically functional insulinomas associated with a syndrome of hyperinsulinism and hypoglycemia caused neurologic symptoms such as episodic seizures, weakness, ataxia, mental confusion, depression and collapse $[1,4,6-10,15,16]$. These clinical signs reflect both inadequate glucose supply to the central nervous system and increased catecholamine secretion by the adrenal medulla in response to hypoglycemia $[8-11,15,16]$. Uncommon manifestations of insulinomas in dogs include weight gain, due to the anabolic effects of insulin, and persistent insomnia. In a minority of affected animals, peripheral polyneuropathy develops and is characterized by demyelination, remyelination, and, to a lesser extent, axonal degeneration [1].

Aggressive behavior of insulin secreted tumors is diagnosed by their histological appearance, in malignant cases the cells may be less uniform in size and shape than in benign ones. Demonstration of vascular or lymphatic invasion, the most important criterion of malignancy, may require examination of multiple sections. Adenomas are slow-growing, sharply delineated, and encapsulated, and are amenable to surgical excision [1].

The aim of this study was to present, a case of malignant insulinoma with multiorgan metastasis in a dog suffer from constipation and inappetence that previously not documented clinical symptom as insulinomas were evaluated by clinical, cytological, histopathological and immunohistochemical findings.

\section{CASE}

A 7-year-old hunting dog presented to the clinic with complaints of constipation and inappetence during for 4 days. Clinical efforts not solved the constipation. After clinical examination dog was decided to laparotomy. During the operation multiple masses were observed in liver, lymph nodes and spleen. A big tumoral mass that caused the adhesions at gut was observed in pancreas. Because of the poor prognosis the dog was euthanatized and necropsy performed. During the necropsy in addition to liver, lymph nodes and spleen multiple tumoral masses were also observed at lungs. Impression smears were prepared from the tumoral mass and stained with Giemsa method. Tissue samples from tumoral mass and all visceral organs were collected and fixed $10 \%$ buffered formalin for histopathological examination. After routine pathology procedure tissue samples embedded the paraffin and cut 5 $\mu \mathrm{m}$ thicknesses. Sections stained with hematoxylin-eosin and examined under light microscope. After histopathological examination selected tissue sections were then immunostained with insulin antiserum (Anti-insulin+ Proinsulin antibody, [D6D4] (ab8304), 1/100 dilution) ${ }^{1}$ and glucagon antiserum (Anti-glucagon antibody, (ab8055), 1/100 dilution) ${ }^{1}$ according the manufacturer's instructions using a routine streptavidin- biotin peroxidase technique. Streptavidin-biotin immunoenzymatic antigen detection system [EXPOSE Mouse and Rabbit Specific HRP/DAB Detection IHC kit (ab80436) $]^{1}$ was used as a second antibody. The antigens were demonstrated by using diaminobenzidine (DAB) as the chromogen.

Clinical examination revealed severe abdominal distension and mild respiratory distress. The dog was reluctant to move. Diffuse loss of detail without free air observed radiographically. According to clinical findings and owners' acceptance laparotomy was performed. Because of diffuse multiple macroscopical masses seen after midline abdominal approach euthanasia performed at the same time.

Necropsy revealed, approximately $500 \mathrm{~mL}$ bloody serous fluid in the abdominal cavity. The intestinal walls and mesenterial vessels were hyperemic. Hemorrhagic and necrotic areas were in seen in intestine. A firm, whitishpink color, hemorrhagic and necrotic $9 \times 5 \times 3 \mathrm{~cm}$ in diameter mass was observed on the caudal part of pancreas. Cut surface of the mass was bloody, and big necrotic areas were observed. Mesenterial adhesions were formed due to mass between duodenum and jejunum. Intestinal content full fill the gut lumen before the adhesion area. 


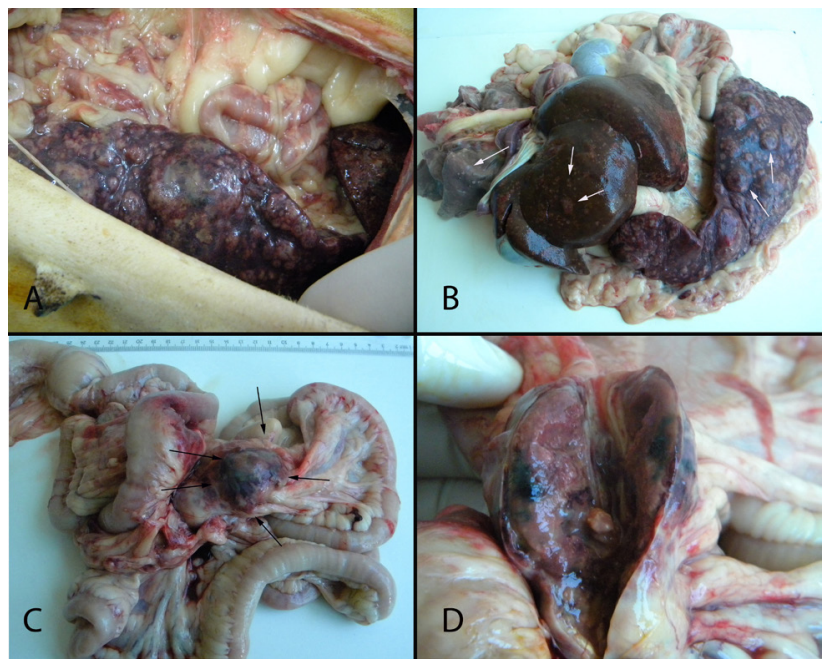

Figure 1. A- Gross appearance of the metastatic tumoral masses during laparotomy. B- Metastatic tumoral masses in liver, spleen and lungs (arrows) after necropsy. C- Gross appearance of the insulinoma (arrows). D- Cut surface of the insulinoma.

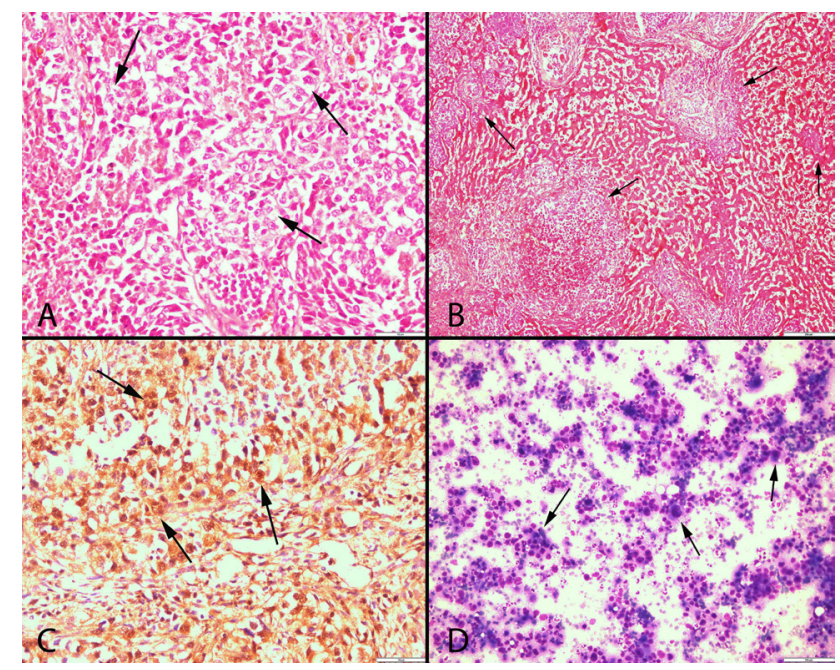

Figure 2. A- Histopathological appearance of the insulinoma and numerous insulin secreted cells (arrows), HE [Bar= $50 \mu \mathrm{m}]$. BLiver metastases of the insulinoma (arrows), HE [Bar= $200 \mu \mathrm{m}]$. C-Insulin positive cells (arrows) by immunohistochemical staining, streptavidin biotin method [Bar= $50 \mu \mathrm{m}]$. D- Impression smear of the tumoral masses and numerous insulin secreted epithelial cells (arrows), Giemsa staining [Bar= $100 \mu \mathrm{m}]$.

At the gross examination, firm, various sized and whitish in color multiple metastatic masses were seen in spleen, liver, lymph nodes and lungs (Figure 1). In addition to multiple metastatic nodules in different organs, kidneys were pale and had degenerative changes.

In cytological preparations of the lesion, epithelial cells in different shapes and sizes were observed. Giemsa stained cells had scant cytoplasm, big irregular nuclei and marked nucleoli. Numerous degenerative and necrotic cells were also noticed in the smear. In addition to tumoral cells marked inflammatory cells mainly composed of neutrophil leukocytes and lymphocytes were observed (Figure 2.D).

At the histopathological examination, sections prepared from pancreatic mass revealed multilobulated tumor nodules composed mainly of epithelial cells arranged in tubuloalveolar and papillary patterns with occasionally lumen formation. The mass had abundant fibrous septa that give lobular appearance to the tissue. The tumor consisted of anaplastic, pleomorphic cells that have granular eosinophilic cytoplasm. These cells had variably sized, irregular nuclei, vesicular and prominent nucleolus. Diffuse necrotic areas were noted in the mass. Higher mitotic activity was observed in both primary tumoral mass and metastatic foci. According the findings histopathological diagnosis of exocrine pancreatic carcinoma was made (Figure 2A-B).

Tumoral cells were strongly positively immunostained with insulin and negative for glucagon. Intensive staining was observed in primary tumor that metastatic masses. Localization of the staining was cytoplasmic and generally diffuse in some cells granular staining was also observed (Figure 2C).

\section{DISCUSSION}

Although pancreatic tumors rarely occur, insulinoma is the most common islet-cell neoplasia in dogs $[1,4,7]$. A definitive diagnosis of insulinoma requires histopathological evidence of a tumor in tissue samples $[6,9,14]$. In this case, a definitive diagnosis of insulinoma were made by cytological, histopathological and immunohistocemical examination.

Clinically functional insulinomas associated with a syndrome of hyperinsulinism, episodic hypoglycemia, and neurologic signs. Signs of sympathetic nervous system stimulation include trembling, muscle fasciculations, restlessness, hunger, and behavioral changes and often precede signs of neuroglycopenia $[1,4]$. In present case, the dog presented to the clinics complains of constipation and in appetence. There was no nervous symptom that thought to the insulinoma.

Most insulinomas are small, measuring $1 \mathrm{~cm}$ or less in diameter, so that a thorough search for a suspected tumor is necessary. Intraoperative identification may be facilitated by intravenous administration of methylene blue that becomes concentrated in the endocrine pancreas [1]. In this case, the tumor was $9 \times 5 \times 3 \mathrm{~cm}$ in diameter and this tumor was very big and metastases of the tumor were numerous. It is possible 
to assess the tumor very aggressive and malign for its pathological findings.

Most insulinomas in animals are generally has malignant behavior and locally invasive Early metastasis to regional lymph nodes and liver commonly observe at diagnosis $[3,10,15,16]$. In addition, metastases to peritoneal surface, other abdominal organs and lung may be seen. Distant metastasis to other organs, such as vertebrae, femur, intracranial, tonsil esophagus and submandibular lymph nodes also documented $[2,4,11]$. Carcinomas may be larger than adenomas and may have a multilobular appearance and contain foci of hemorrhage and necrosis [1]. In agreement in this study with previous studies, our case was very big and aggressive than literature knowledge. Metastases were observed interestingly spleen that very rare site of insulinoma metastases in addition to the lymph nodes, lungs and livers.
Insulinomas are usually diagnosed in middleaged to older dogs, without sex predisposition. Large breeds encountered as predisposed factor but this tumor may occur in any breed $[2,6,8-10,12,14,17]$. We reported insulinoma in a 7-year- old hunting dog with totally agreement previous documented cases.

This study reported a case of malign insulinoma with multiorgan metastasis in a 7-year-old dog that suffer from constipation and in appetence. These clinical symptoms not previously reported in insulinoma cases previously. Although the tumor was very big and aggressive clinical symptoms were unrelated the classical insulinoma cases.

\section{MANUFACTURER}

${ }^{1}$ abcam - Immunohistochemistry (IHC) kits and reagents. Cambridge, UK.

Declaration of interest. The authors report no conflicts of interest. The authors alone are responsible for the content and writing of the paper.

\section{REFERENCES}

1 Capen C.C. 2007. Insulinoma. In: Maxie M.G. (Ed). Jubb, Kennedy and Palmer's Pathology of Domestic Animals. 5th edn. v.2. Philadelphia: Saunders Elsevier, pp.421-423.

2 Chang S.C., Liao J.W., Lin Y.C., Liu C.I. \& Wong M.L. 2007. Pancreatic acinar cell carcinoma with intracranial metastasis in a dog. Journal of Veterinary Medical Science. 69(1): 91-93.

3 Cordner A.P., Sharkey L.C., Armstrong P.J. \& Mcateer K.D. 2015. Cytologic findings and diagnostic yield in 92 dogs undergoing fine-needle aspiration of the pancreas. Journal of Veterinary Diagnostic Investigation. 27(2): 236-240.

4 Cullen J.M. \& Popp J.A. 2002. Tumors of the exocrine pancreas. In: Meuten D.J. (Ed). Tumors in Domestic Animals. 4th edn. Ames: Iowa State University Press, pp.478-481.

5 Dennis M.M., O’Brien T.D., Wayne T., Kiupel M., Williams M. \& Powers B.E. 2008. Hyalinizing pancreatic adenocarcinoma in six dogs. Veterinary Pathology. 45: 475-483.

6 Goutal C.M., Brugmann B.L. \& Ryan K.A. 2012. Insulinoma in dogs: a review. Journal of American Animal Hospital Association. 48: 151-163.

7 Hess R.S. 2010. Insulin-Secreting Islet Cell Neoplasia. In: Ettinger S.J. \& Feldman E.C. (Eds). Textbook of Veterinary Internal Medicine. 7th edn. Philadelphia: W.B. Saunders, pp.1779-1782.

8 Koenig A. 2009. Hypoglycemia. In: Silverstein D.C. \& Hopper K. (Eds). Small Animal Critical Care Medicine. Philadelphia: W.B. Saunders, pp.295-299.

9 Kruth S.A., Feldman E.C. \& Kennedy P.C. 1982. Insulin-secreting islet cell tumors: establishing a diagnosis and the clinical course for 25 dogs. Journal of American Veterinary Medical Association. 181: 54-58.

10 Leifer C.E., Peterson M.E. \& Matus R.E. 1986. Insulin-secreting tumor: diagnosis and medical and surgical treatment in 55 dogs. Journal of American Veterinary Medical Association. 188: 60-64.

11 Morris J. \& Dobson J. 2001. Gastro-intestinal Tract. In: Morris J. \& Dobson J. (Eds). Small Animal Oncology. Oxford: Blackwell Science Ltd., pp.125-143.

12 Nelson R.W. \& Couto C.G. 1998. Insulinoma. In: Nelson R.W. \& Couto C.G. (Eds.). Small Animal Internal Medicine. 2nd edn. Missouri: Mosby Inc., pp.734-774.

13 Oskoui-Zadeh K., Jamshidi S.H., Ashrafihelan J. \& Veshkini A. 2008. Exocrine pancreatic adenocarcinoma in a toy breed dog. Iranian Journal of Veterinary Research Shiraz University. 9(1): 87-91.

14 Polton G.A., White R.N., Brearley M.J. \& Eastwood J.M. 2007. Improved survival in a retrospective cohort of 28 dogs with insulinoma. Journal of Small Animal Practice. 48: 151-156. 
15 Tobin R.L., Nelson R.W., Lucroy M.D., Woolridge J.D. \& Feldman E.C. 1999. Outcome of surgical versus medical treatment of dogs with beta cell neoplasia: 39 cases (1990-1997). Journal of American Veterinary Medical Association. 215: 226-230.

16 Trifonidou M.A., Kirpenstiin J. \& Robben J.H. 1998. A retrospective evaluation of 51 dogs with insulinoma. Veterinary Quarterly. 20: 114-115.

17 Van Ham L., Braund K.G., Roels S. \& Putcuyps I. 1997. Treatment of a dog with an insulinoma-related peripheral polyneuropathy with corticosteroids. Veterinary Record. 141: 98-100. 